\title{
Modification of waste polyacrylonitrile fiber and its application as a filtrate reducer for drilling
}

\author{
Pei-Zhi Yu ${ }^{1}$
}

Received: 30 June 2014/ Published online: 28 March 2015

(c) The Author(s) 2015. This article is published with open access at Springerlink.com

\begin{abstract}
Cationic polymer fluid loss additive (CPFL) was prepared by using the reaction of 2,3-epoxypropyltrimethyl ammonium chloride (EPTMAC) (as cationic reagent) with the amide group in the molecular structure of the sodium salt of partially hydrolyzed polyacrylonitrile fibers (HPAN-Na). The chemical reaction was determined by studying the infrared absorption peaks of the materials and the products. The results proved that the cationic groups of EPTMAC were successfully grafted onto the HPAN molecular chain. The composition of the molecular chain of the product CPFL was determined by investigation and calculation of the elemental analysis results of the grafted HPAN and the final reaction product CPFL. The drilling fluid performance was evaluated, and the result showed that when the cation content was more than $0.27 \mathrm{mmol} / \mathrm{g}$, the drilling fluid would have good resistance to fluid loss and to pollution from calcium chloride.
\end{abstract}

Keywords Acrylic fiber - Cationic agents - Fluid loss agent $\cdot$ Spectroscopy

\section{Introduction}

In oil-drilling operations, a filtrate reducer is the most commonly used drilling fluid additive. Its main functions are reducing drilling fluid filtration by forming thin and

Pei-Zhi Yu

yupz@cugb.edu.cn

1 School of Engineering and Technology, China University of Geosciences (Beijing), Beijing 100083, China

Edited by Xiu-Qin Zhu dense filter cakes on the wellbore, thus reducing the mutual permeability between the mud filtrate and wellbore, minimizing wellbore instability, and ensuring the stability of drilling fluid performance (Zhang and Deng 1998). Partially hydrolyzed sodium polyacrylonitrile with an anionic hydration group, polyacrylonitrile calcium salts, and polyacrylonitrile ammonium salts are widely used as filtrate reducers, which meet the requirements of well drilling operations below $120^{\circ} \mathrm{C}$ (Cui and Zhu 1999; Ahmed et al. 2013). The application of an allyl copolymer with cationic groups in the drilling fluid has been reported. This mainly revolves around its application to cationic and zwitterionic drilling fluid systems (Zhang and Deng 1998; Warren et al. 2000). Hydrophobically modified polyacrylamide as a sealing agent has been used in water-based drilling fluid. This can effectively improve the temperature and salt resistance of water-based drilling fluid system (Xie et al. 2013). Lubricants based on water-soluble polyacrylamide have also been used in drilling, particularly in low-density drilling fluid systems (Wang 2013). In recent years, standards of drilling fluid systems have become higher with the increase in deep and complex wells. Therefore, a filtrate reducer containing cationic groups is believed to have a promising application (Zlotnikov et al. 2006). There have been reports about acrylic waste fiber that can enable the drilling fluid loss agent to be cationically grafted under alkaline conditions (Yu et al. 2004; Celik et al. 2012). Still missing are the molecular structure of the cationic agent and the cationic degree of the final products. The characterization of the structure of such reaction products is yet to be reported. The application of the products and their mechanisms of activity in the drilling fluid should be further investigated. 


\section{Synthesis of cationic fluid loss additive}

\subsection{Materials and instruments}

Industrial by-product waste polyacrylonitrile fiber was provided by the Anqing Chemical Fiber Factory (China). AR $\mathrm{NaOH}$, AR 2,3-epoxypropyltrimethyl ammonium chloride (EPTMAC), and CR Ethanol were provided by Sinopharm Chemical Reagent Beijing Co., Ltd.

Instrumentation included a reflux reaction device; API (American Petroleum Institute) filtration press; high-speed mixer; a Magna-IR 750 Fourier transform infrared spectrometer (Thermo Nicolet Corporation, USA); and a Vario EL element analyzer (Elementar Analysensysteme GmbH, Germany).

\subsection{Chemical reaction principle}

Cationic fluid loss additive was prepared by reactions of EPTMAC with amide groups, catalyzed by alkali (Tang et al. 1995; Ma and Zhao 1995). The principle of the chemical reaction is shown in Fig. 1.

\subsection{Synthesis of cationic fluid loss additive}

A total of $15 \mathrm{~g}$ of partially hydrolyzed polyacrylonitrile sodium salt (HPAN) and $150 \mathrm{~mL}$ of distilled water were added to a $250 \mathrm{~mL}$ three-necked flask, mixed, and then heated to $70-80{ }^{\circ} \mathrm{C}$ in a water bath. After that, a cationic additive 2,3-epoxypropyltrimethyl ammonium chloride solution with a mass ratio of $20 \%-50 \%$ based on HPAN was added and stirred at constant temperature for three hours.

The reaction product was washed three times with ethanol to obtain viscous products which were dried at $105 \pm 5{ }^{\circ} \mathrm{C}$ in an oven, and then pulverized to obtain cationic product CPFL with a cationic degree of $0.2-0.5 \mathrm{~m}$ $\mathrm{mol} / \mathrm{g}$.

\section{Structural characterization of cationic fluid loss additives}

Acrylic waste fiber, hydrolysis products HPAN, and cationic product CPFL (with a cationic degree of $0.49 \mathrm{mmol} /$ $\mathrm{g}$ determined by chemical titration) were tested by Fourier transform infrared spectroscopy. The graft substrate HPAN and cationic fluid loss additive CPFL were characterized by elemental analysis to determine the composition of their molecular chains.

\subsection{IR spectrum}

Figure 2 shows the infrared spectrum of acrylic waste fiber PAN, partially hydrolyzed polyacrylonitrile sodium HPAN, and fluid loss agent CPFL.

In the infrared spectrum of acrylic waste fiber PAN, the stretching vibration peak of nitrile group was at
Fig. 1 Schematic diagram of the fluid loss agent made by using cationic acrylic fiber waste a Hydrolysis of acrylic waste fiber, b Cationic agent

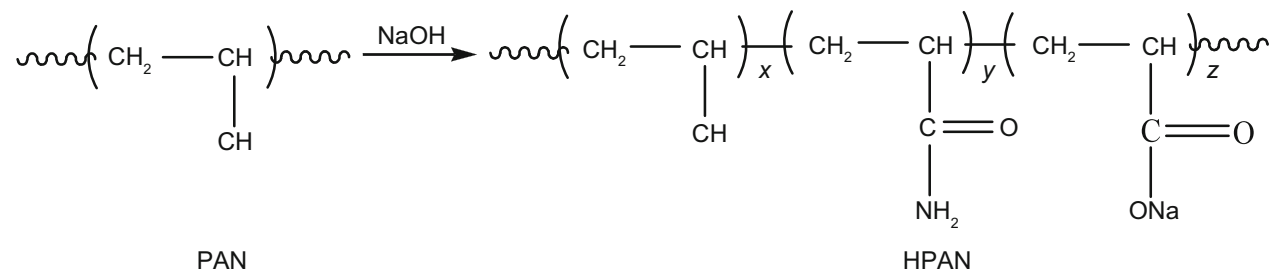

(a)

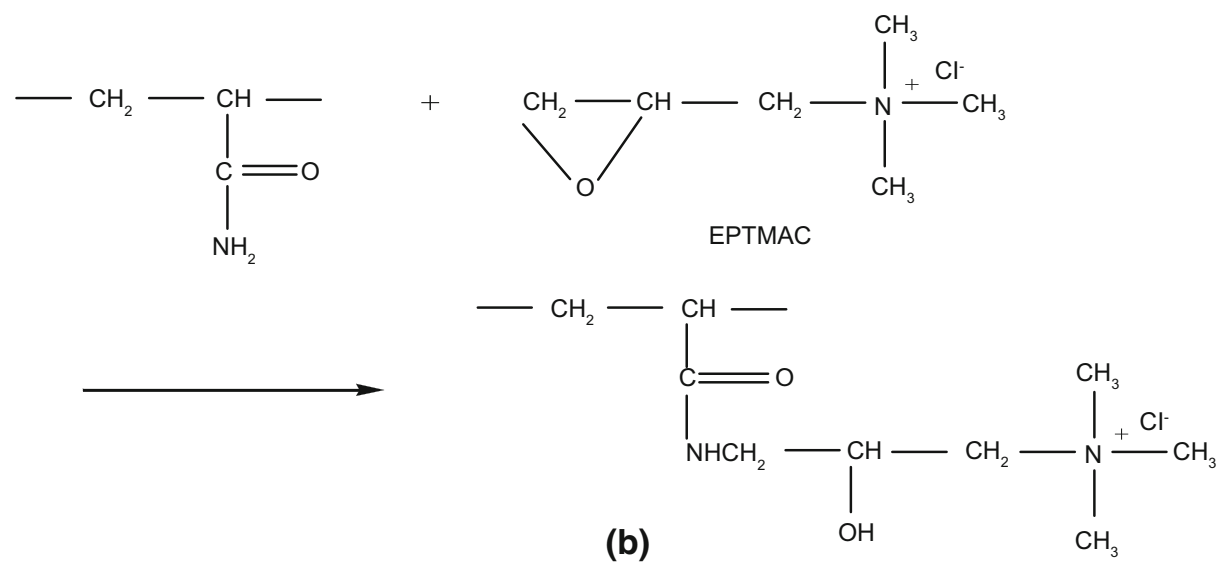




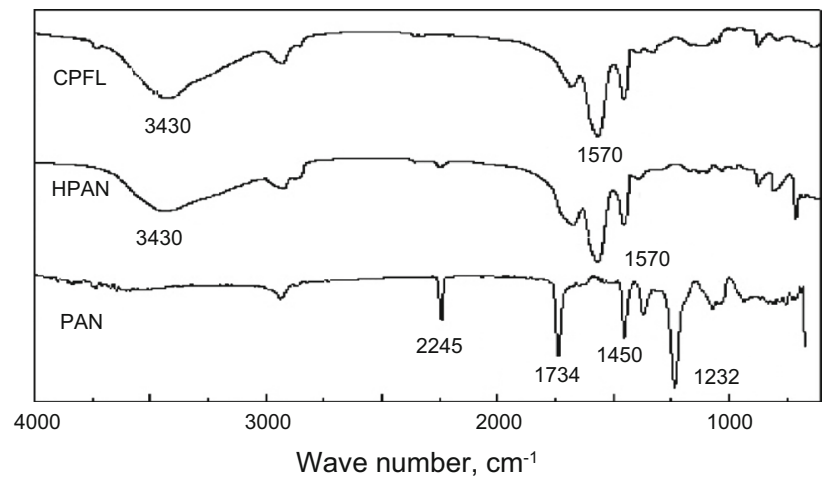

Fig. 2 FTIR of raw materials and products

$2245 \mathrm{~cm}^{-1}$. The peak at $1450 \mathrm{~cm}^{-1}$ was assigned to the variable angle vibration of methylene. These were the characteristic absorption peaks of polyacrylonitrile and there was a strong absorption peak at $1734 \mathrm{~cm}^{-1}$, which was the unique stretching vibration absorption peak of carbonyl groups. It indicated that the raw material contained acrylate, the copolymer of acrylonitrile and acrylicester.

In the infrared spectrum of partially hydrolyzed sodium polyacrylonitrile (HPAN), which was boiled for two hours for base-catalyzed hydrolysis at atmospheric pressure, the absorption peak of nitrile group at $2245 \mathrm{~cm}^{-1}$ became very weak, indicating that the vast majority of the nitrile groups were hydrolyzed to amide and carboxyl groups. The amide carbonyl stretching vibration peak was at $1675 \mathrm{~cm}^{-1}$; the $\mathrm{N}-\mathrm{H}$ stretching vibration absorption peak appeared at $3431 \mathrm{~cm}^{-1}$ and the characteristic peaks of -COO- appeared at 1570 and $1400 \mathrm{~cm}^{-1}$.

The infrared spectrum of cationic filtrate reducer CPFL (Fig. 2) showed that nitrile peak was very weak after the HPAN cationic reaction, indicating that nitrile was hydrolyzed in the cationic process. A pair of peaks appeared at 1415 and $1326 \mathrm{~cm}^{-1}$, which are the characteristic absorption peaks of secondary alcohol, indicating that the epoxy ring was opened, and cationic groups were successfully grafted to HPAN chains.

\subsection{Elemental analysis}

The elemental analysis results of partially hydrolyzed polyacrylonitrile sodium (HPAN) and its cationic products CPFL are shown in Table 1.

Table 1 Elemental analysis results of HPAN and CPFL
According to the structural formula of the hydrolysis product of acrylic fiber and its cationic products ( $\mathrm{Li}$ and Zhang 1999), the elemental concentration of C, N, and O can be calculated with the following equations:

Cationic product CPFL:

$C(\%)=12 \times(3 x+3 y+9 z)$

$N(\%)=14 \times(x+2 z)$

$O(\%)=16 \times(x+2 y+2 z)$

Deduction: $x=\frac{O}{16}-\frac{C}{18}+\frac{N}{7}$

$y=\frac{O}{32}-\frac{N}{28}$

$z=\frac{C}{36}-\frac{O}{32}-\frac{N}{28}$

With $C=38.05, N=2.49$, and $O=29.54$, we obtained $x=0.088, y=0.834$, and $z=0.045$. The molar ratio of the three structural units was 9.10/86.2/4.65. The molar masses for each of the structure units were 71,94 , and $222.5 \mathrm{~g} / \mathrm{mol}$, respectively.

Among them, the $x, y$, and $z$ represent the number of chains in three different structure units of HPAN molecular chain, as shown in Fig. 1.

Cationic degree of cationic polymer filtrate reducer CPFL:

$$
\begin{aligned}
& 0.045 \times 10^{3} \\
& 71 \times 0.088+94 \times 0.834+222.5 \times 0.045 \\
& =0.475(\mathrm{mmol} / \mathrm{g})
\end{aligned}
$$

The calculation from elemental analysis agreed well with the titration data of $0.49 \mathrm{mmol} / \mathrm{g}$. The result of the elemental analysis further demonstrated that the cationic reagent was successfully grafted onto the molecular chain of the partially hydrolyzed polyacrylonitrile sodium salt.

\section{Evaluation of drilling fluid performance}

\subsection{Influence of cationic degree on drilling fluid filtration}

The base drilling fluid was prepared as follows: $100 \mathrm{~g}$ of bentonite and $8 \mathrm{~g}$ of anhydrous $\mathrm{Na}_{2} \mathrm{CO}_{3}$ were added to $1000 \mathrm{~mL}$ of water, and stirred for about $20 \mathrm{~min}$ with a high-speed mixer, and then placed under a sealed condition for about $24 \mathrm{~h}$ at room temperature. The property of the drilling fluid was then determined according to the API standard.

CPFL sample (with a concentration of 0.5 or $1.0 \mathrm{wt} \%$ ) with different cationic degrees was added to the base drilling fluid. The drilling fluid property was tested after 
Table 2 Influence of cationic degree on drilling fluid filtration

\begin{tabular}{|c|c|c|c|c|c|}
\hline Concentration of CPFL (wt\%) & Cationic degree, $\mathrm{mmol} / \mathrm{g}$ & $\mathrm{AV}, \mathrm{mPa} \mathrm{s}$ & $\mathrm{PV}, \mathrm{mPa} \mathrm{s}$ & $\mathrm{YP}, \mathrm{Pa}$ & FL, mL \\
\hline \multirow[t]{5}{*}{0.5} & 0.00 & 6.5 & 6.5 & 0.0 & 6.9 \\
\hline & 0.27 & 8.8 & 7.5 & 1.3 & 5.2 \\
\hline & 0.47 & 9.0 & 7.0 & 2.0 & 5.5 \\
\hline & 0.54 & 9.0 & 7.0 & 2.0 & 6.0 \\
\hline & 1.03 & 11.0 & 6.0 & 5.0 & 9.0 \\
\hline \multirow[t]{3}{*}{1.0} & 0.00 & 12.0 & 11.0 & 1.0 & 4.6 \\
\hline & 0.27 & 17.0 & 14.0 & 3.0 & 3.2 \\
\hline & 0.54 & 15.5 & 12.0 & 3.5 & 4.0 \\
\hline
\end{tabular}

$A V$ apparent viscosity, $P V$ plastic viscosity, $Y P$ yield point, $F L$ filtration

20 min of stirring at a high rate. The results are shown in Table 2.

Table 2 shows that the drilling fluid filtration decreased with the increase in cationic degree. The CPFL product had a good filter reducing effect in the cationic degree ranging from 0.27 to $0.54 \mathrm{mmol} / \mathrm{g}$. Drilling fluid filtration achieved a minimum at a cationic degree of $0.27 \mathrm{mmol} / \mathrm{g}$.

\subsection{Influence of cationic degree on drilling fluid salt resistance}

A total of $1.0 \%$ of CPFL sample with different cationic degrees was added to the base drilling mud containing $10 \%$ of bentonite and stirred for $20 \mathrm{~min}$, and then contaminated using $15 \%$ of $\mathrm{NaCl}$, after that $\mathrm{CaCl}_{2}$ with different mass fractions of $1 \%, 2 \%$ or $3 \%$ was added. After $20 \mathrm{~min}$ of stirring, the performance of the mixed drilling fluid system was evaluated, and the influence of the cationic degree on the performance of contaminated drilling fluids was measured as shown in Fig. 3.

The results showed that for the drilling fluid system contaminated by $\mathrm{NaCl}$ and $\mathrm{CaCl}_{2}$ salts, the API filtration

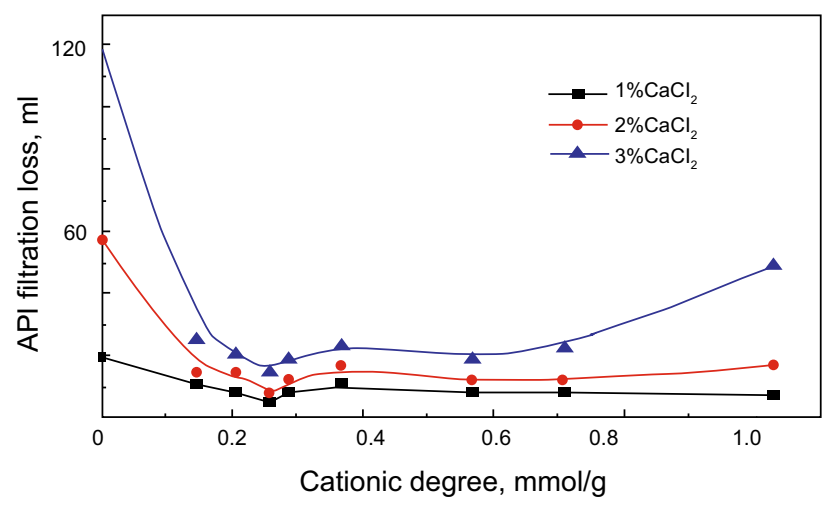

Fig. 3 Relationship between cationic degree and drilling fluid filtration loss at different concentrations of $\mathrm{CaCl}_{2}$ loss would increase as the $\mathrm{CaCl}_{2}$ concentration increased. When cationic degree reached $0.15 \mathrm{mmol} / \mathrm{g}$, the drilling fluid filtration loss had a sharp decline; at the cationic degree of $0.27 \mathrm{mmol} / \mathrm{g}$, it had its best fluid loss effect.

\subsection{Analysis of action mechanism of cationic filtration reducer}

The electrostatic attraction between anionic groups and cationic groups was enhanced by the addition of cations to the molecular chain of partially hydrolyzed sodium polyacrylonitrile, which in turn further enhances the electrostatic attraction between filtration reducer cationic groups and clay particle anionic groups. Therefore, a network structure was formed to a certain extent, and could improve the polymer's resistance to salt pollution and its ability to confine free water. The filtration-reducing effect significantly improved (Wang and Zhang 1995). But when the cationic degree is too big, with the enhancement of the inhibitory effect of cationic quaternary ammonium, flocculation capacity is too strong, the solid particles in the system become excessively big, the number of colloidal microparticles reduces, the density of filter cake becomes small, so the permeability increases, resulting in greater filtration.

\section{Conclusion}

Partially hydrolyzed sodium polyacrylonitrile was prepared through the hydrolytic reaction of acrylic fiber under alkaline conditions, which grafted cationic monomer to synthesize cation-modified polyacrylonitrile. The synthetic modification process is simple, easy to control, and can synthesize good drilling fluid loss additive cationic products. The characterization of the product molecular structure and the drilling fluid application performance indicated that if the cationic reaction and the cationic degree could be properly controlled, it would be applicable as 
a drilling fluid loss agent, and have good resistance to calcium chloride contamination.

Acknowledgments This work was supported by China National High Technology Research and Development Program (863 Program, 2013AA064803).

Open Access This article is distributed under the terms of the Creative Commons Attribution License which permits any use, distribution, and reproduction in any medium, provided the original author(s) and the source are credited.

\section{References}

Ahmed MA, Ahmed AK, Mahmoud IA, et al. Investigation of some copolymers based on acrylic salts as circulation loss control agents. Egypt J Pet. 2013;22(4):481-91.

Cui YM, Zhu LJ. Study on the new technique for producing $\mathrm{Na}$ HPAN. Spec Petrochem. 1999;9:26-8.

Celik M, Qudrat ML, Akyuz E, et al. Synthesis and characterization of acrylic fibers-g-polyacrylamide. Fibers Polym. 2012;13(2):145-52.

Li G, Zhang SM. The synthesis and application of cationic polyelectrolyte with high density charge. China Environ Sci. 1999;2:145-8.

Ma XP, Zhao JB, Wang WX, et al. Acrylamide/trimethyl allyl ammonium chloride copolymer as heat and salts tolerant clay stabilizer: synthesis and properties. Oilfield Chem. 1995;12(3):197-200.

Tang SZ, Hu XQ, Ma XP, et al. Synthesis of monomer of $N, N$ dimethyl hydroxyethyl acrylamide and the preparation of its cationic copolymer with acrylamide. J Southwest Pet Inst. 1995;17(4):101-9.

Warren B, Van der Horst PM, Van T Zelfde TA. Quaternary nitrogen containing amphoteric water soluble polymers and their use in drilling fluids. 2000 WO: 0060023.

Wang $\mathrm{ZH}$. The development overview on the domestic drilling fluids and drilling fluid additives. Sino-Glob Energy. 2013;18(10):34-41.

Wang B, Zhang Y. Application of small cationic polymer drilling fluid in Mao Ping 1 well deviated. Pet Drill Tech. 1995;23(1):27-9.

Xie BQ, Qiu ZS, Cao J. A novel hydrophobically modified polyacrylamide as a sealing agent in water-based drilling fluid. Pet Sci Technol. 2013;31(18):1866-72.

Yu PZ, Wang XY, Geng TM, et al. Study of cationic modified polyacrylonitrile waste fibers used in cationic polymer drilling fluid. Mod Chem Ind. 2004;24(10):31-4, 36.

Zhang JG, Deng H. Filtrate reducer with temperature and salt resistance used in MMH drilling fluid. Drill Fluid Complet Fluid. 1998;15(2):36-7.

Zlotnikov II, Khilo PA, Zubritskii MI. Polymer-based lubricating fluids for mechanical treatment of glass. J Frict Wear. 2006;27(6):63-5. 